\title{
DIVERSITY OF ALGAE SPECIES IN XUANHUONG LAKE, DALAT
}

\author{
Nguyen Thi Thuy Linh" ${ }^{a^{*}}$, Le Ha Thu
}

${ }^{a}$ The Faculty of Biology, Dalat University, Lamdong, Vietnam

\section{Article history}

Received: June $02^{\text {nd }}, 2016$

Received in revised form ( $\left.1^{\text {st }}\right)$ : July 02 $2^{\text {nd }}, 2016 \mid$ Received in revised form $\left(2^{\text {nd }}\right)$ : August $02^{\text {nd }}, 2016$ Accepted: August $28^{\text {th }}, 2016$

\begin{abstract}
Xuanhuong Lake is considered the heart of Dalat. In this study, algal abundance and diversity in Xuanhuong Lake were studied. Water samples were collected from four different sampling sites from September 2012 to January 2013. The identified species were 75 species belonging to 13 orders, 8 classes, 5 phyla (Cyanophyta, Chlorophyta, Bacillatoriophyta, Euglenophyta, Pyrrhophyta). Chlorophyta has the most species with 33 species (44\%), the next was Bacillatoriophyta with 18 species (24\%), Cyanophyta with 12 species (16\%), Euglenophyta with 11 species (15\%), and the least was Pyrrophyta: 1 species (1\%). There has also been bioindicator species including Euglena viridis, Euglena acus, and Synedra ulna. Therefore, Xuanhuong Lake is considered mesosaprobic
\end{abstract}

Keywords: Bioindicator; Chlorophyta; Diversity; Xuanhuong Lake.

\section{INTRODUCTION}

Xuanhuong Lake is an artificial lake located in the center of Dalat city, which is closely connected to the history of Dalat city.

Xuanhuong Lake has an area of 32 ha and an average depth of $1.5 \mathrm{~m}-3.5 \mathrm{~m}$. It is located on an altitude of $1,478 \mathrm{~m}$ above sea level with a capacity of approximately 7.2 million $\mathrm{m}^{3}$. Xuanhuong Lake's perimeter is around $5 \mathrm{~km}$; it has a crescent shape with many beautiful scenes alongside, such as $\mathrm{Cu}$ Hill, Yersin Park, and City Flower Garden. The water quality in the lake depends on the upper reach and watershed. Most of the waste water from tourism, business and agricultural activities is accumulated here.

In recent years, there have been several algal blooms in Xuanhuong Lake which have caused harmful influences on local people's life and tourism. The government and scientists have already organized various conferences to discuss the issue of water 
pollution regarding renovation of sedimentation tank, building bar screens to prevent large objects like cans, rags, sticks, plastic packets etc. to enter the sewage system, growing water hyacinth in the sedimentation tanks, etc. However, most studies have just focused on finding out the cause of the bloom or the algae species. There has been no research on the algae overview of Xuanhuong Lake to help people understand the relationships among species, biotic and abiotic factors as well as the energy and material cycle in lakes, especially the use of the local algae species to treat water pollution and limit algae bloom to help build an efficient and sustainable solution.

\section{MATERIALS AND METHODS}

\subsection{Locations and time of sampling}

- Time: from September 2012 to January 2013; 1 time/month.

- Locations: at the 4 positions located by GPS with the following coordinates:

\begin{tabular}{llll}
\multicolumn{4}{c}{ Table 1. Coordinates and locations of sampling } \\
\hline Locations & $\begin{array}{l}\text { Symbol of } \\
\text { sampling } \\
\text { locations }\end{array}$ & $\begin{array}{l}\text { Coordinates } \\
\text { North latitude }\end{array}$ & East longitude \\
\hline Near Causat & XHS1 & $11^{\circ} 56^{\prime} 52^{\prime \prime}$ & $108^{\circ} 27^{\prime} 6^{\prime \prime}$ \\
Bichcau Lake & XHS2 & $11^{\circ} 56^{\prime} 21^{\prime \prime}$ & $108^{\circ} 26^{\prime} 21^{\prime \prime}$ \\
Thanhthuy & XHS3 & $11^{\circ} 56^{\prime} 21^{\prime \prime}$ & $108^{\circ} 26^{\prime} 16^{\prime \prime}$ \\
Middle lake & XHS4 & $11^{\circ} 56^{\prime} 22^{\prime \prime}$ & $108^{\circ} 27^{\prime} 7^{\prime \prime}$ \\
\hline
\end{tabular}

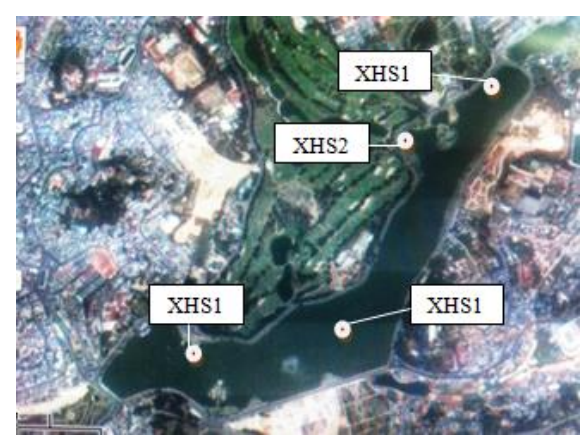

Figure 1. Locations of sampling in Xuanhuong Lake

\subsection{Collection and handling of samples}

Samples of floating algae were collected with plankton nets N0. 64, N0. 70, N0. 75. Algae growing on surfaces were collected by hand. 
The samples were fixed in $4 \%$ formaldehyde. The method of Nguyễn (2003) was used in the whole process.

\subsection{Analysis sample and identification}

Samples were analyzed at the laboratory of the Faculty of Biology, Dalat University with Cole Parmer, National Digital DC5-163 microscope.

Identification of samples is based on the morphology and classification criteria by Phạm (1972), Dương (1996), Dương and Võ (1997), Nguyễn (2003), Prescott (1962), Cole and Sheath (1990), Smith (1950) and Yamaghishi (1992).

\section{RESULTS AND DICUSSION}

75 species / subspecies of 13 orders, 8 Classes, 5 Phyla: Cyanophyta, Chlorophyta, Bacillatoriophyta, Euglenophyta, Pyrrhophyta (Table 2) have been identified.

Table 2. Species of microalgae at Xuanhuong Lake

\begin{tabular}{|c|c|c|c|c|}
\hline STT & Taxon & Order & Class & Phylum \\
\hline 1 & Staurastrum paradoxum Meyen & Desmidiales & Conjugatophycaea & Chlorophyta \\
\hline 2 & Closterium porrectum Nordst. & - & - & - \\
\hline 3 & Pleurotaenium sp. & - & - & - \\
\hline 4 & Staurastrum gracile Ralfs., & - & - & - \\
\hline 5 & Cosmarium sp & - & - & - \\
\hline 6 & Closterium tumidum Johns & - & - & - \\
\hline 7 & Spirogyra crassa Kuetz. & Zygnematales & - & - \\
\hline 8 & Actinastrum sp. & Chlorococcales & Chlorophyceae & - \\
\hline 9 & Dictyosphaerium Ehrenbergii Naeg & - & - & - \\
\hline 10 & Pediastrum duplex Meyen & - & - & - \\
\hline 11 & Pediastrum obtusum Lucks & - & - & - \\
\hline 12 & $\begin{array}{l}\text { Pediastrum simplex var. } \\
\text { duodenarium (Bailey) Rabenhorst }\end{array}$ & - & - & - \\
\hline 13 & Coelastrum microporum Naeg. & - & - & - \\
\hline 14 & Coelastrum sp. & - & - & - \\
\hline
\end{tabular}


Table 2. Species of microalgae at Xuanhuong Lake (cont.)

\begin{tabular}{|c|c|c|c|c|}
\hline STT & Taxon & Order & Class & Phylum \\
\hline 15 & Actinastrum Hantzshii Lagerh. & - & - & - \\
\hline 16 & Tetrastrum glabrum Alstr.et Tiffany & - & - & - \\
\hline 17 & Tetrastrum multisetum Chod. & - & - & - \\
\hline 18 & Scenedesmus bijugatus Kuetz. & - & - & - \\
\hline 19 & Scenedesmus denticulatus Lagerh. & - & - & - \\
\hline 20 & Scenedesmus acuminatus Chodat & - & - & - \\
\hline 21 & $\begin{array}{l}\text { Scenedesmus dimorphus (Turp.) } \\
\text { Kuetz. }\end{array}$ & - & - & - \\
\hline 22 & $\begin{array}{l}\text { Scenedesmus quadricauda var. } \\
\text { armatus (Chodat.) Dedus., }\end{array}$ & - & - & - \\
\hline 23 & $\begin{array}{l}\text { Scenedesmus pseudoarmatus var. } \\
\text { bicaudatus Hortob. }\end{array}$ & - & - & - \\
\hline 24 & Crucigenia quadrata Morren. & - & - & - \\
\hline 25 & $\begin{array}{l}\text { Tetraedron lobatum var } \\
\text { subtetraedricum Reinsch. }\end{array}$ & - & - & - \\
\hline 26 & Ankistrodesmus sp. & - & - & - \\
\hline 27 & $\begin{array}{l}\text { Ankistrodesmus fusiformis corda } \\
\text { sensu Kors. }\end{array}$ & - & - & - \\
\hline 28 & $\begin{array}{l}\text { Ankistrodesmus falcatus var } \\
\text { spirilliformis (W.et G.S.West) G.S. } \\
\text { West }\end{array}$ & - & - & - \\
\hline 29 & Chlorella luteoviridis Chod. & - & - & - \\
\hline 30 & Chlorella sp. & - & - & - \\
\hline 31 & Ulothrix sp. & Ulotrichales & - & - \\
\hline 32 & Pandorina morum Bory. & Volvocales & - & - \\
\hline 33 & Gonium pectorale Mull & - & - & - \\
\hline 34 & Melosira granulate sp & Discinales & Centricae & Bacillariophyta \\
\hline 35 & Cyclotella sp. & - & - & - \\
\hline 36 & Stephanodiscus sp. & - & - & - \\
\hline 37 & Synedra acus Kuetz. & Araphinales & Pennatae & - \\
\hline 38 & Achnanthes exigua Grun. & Raphinales & - & - \\
\hline 39 & Achnanthes sp. & - & - & - \\
\hline 40 & Cymbella sp. & - & - & - \\
\hline 41 & Gomphonema longiceps Ehr. & - & - & - \\
\hline 42 & Gomphonema parvulum sp & - & - & - \\
\hline 43 & $\begin{array}{l}\text { Gomphonema brasiliense var. } \\
\text { demararae Grun. }\end{array}$ & - & - & - \\
\hline
\end{tabular}


Table 2. Species of microalgae at Xuanhuong Lake (cont.)

\begin{tabular}{|c|c|c|c|c|}
\hline STT & Taxon & Order & Class & Phylum \\
\hline 44 & $\begin{array}{l}\text { Navicula palaisiensis var. lanceolata } \\
\text { Grun. }\end{array}$ & - & - & - \\
\hline 45 & Pinnularia major (Kutz.) $\mathrm{Cl}$. & - & - & - \\
\hline 46 & Pinnularia nobilis Ehr. & - & - & - \\
\hline 47 & Pinnularia microstauron (Ehr.) $\mathrm{Cl}$. & - & - & - \\
\hline 48 & Pinnularia sp. & - & - & - \\
\hline 49 & Hantzschia amphiosys (Ehr.) Grun. & - & - & - \\
\hline 50 & Nitzschia sublinearis Hust & - & - & - \\
\hline 51 & Nitzschia sp. & - & - & - \\
\hline 52 & Merismopedia tenuissima Lemm. & Chroococcales & Chroococceae & Cyanophyta \\
\hline 53 & Tetrapedia sp. & - & - & - \\
\hline 54 & Microcystic aeruginosa Kutz & - & - & - \\
\hline 55 & $\begin{array}{l}\text { Microcystic aeruginosa f. flosaquae } \\
\text { Elenk. }\end{array}$ & - & - & - \\
\hline 56 & Annabaena sp. & Nostocales & - & - \\
\hline 57 & Calothrix sp & - & - & - \\
\hline 58 & Oscillatoria simplicisima Gom. & Oscillatoriales & Hormogoneae & - \\
\hline 59 & Oscillatoria $\mathrm{sp}_{1}$ & - & - & - \\
\hline 60 & Oscilatoria $\mathrm{sp}_{2}$ & - & - & - \\
\hline 61 & Lyngbya $\mathrm{sp}_{1}$ & - & - & - \\
\hline 62 & Lyngbya $\mathrm{sp}_{2}$ & - & - & - \\
\hline 63 & Phormidium fragile (Menegh.) Gom. & - & - & - \\
\hline 64 & Euglena acus Ehr. & Euglenales & Euglenophyceae & Euglenophyta \\
\hline 65 & $\begin{array}{l}\text { Euglena limnophila var. swirencoi } \\
\text { (Arn.) Popova }\end{array}$ & - & - & - \\
\hline 66 & Euglena oxyuris sp. & - & - & - \\
\hline 67 & Euglena spirogyra sp. & - & - & - \\
\hline 68 & Euglena viridis Ehr. & - & - & - \\
\hline 69 & Phacus caudatus Hubner & - & - & - \\
\hline 70 & Phacus longicauda sp. & - & - & - \\
\hline 71 & Phacus pleuronectes (Ehr.) Duj. & - & - & - \\
\hline 72 & Trachelomonas hispida sp. & - & - & - \\
\hline 73 & Trachelomonas planctonica sp. & - & - & - \\
\hline 74 & Trachelomonas volvocina sp. & - & - & - \\
\hline 75 & $\begin{array}{l}\text { Ceratium hidrundinella (O.F.Muell.) } \\
\text { Dujardin }\end{array}$ & Peridiniales & Dinophyceae & Pyrrhophyta \\
\hline
\end{tabular}


Among 5 phyla, Chlorophyta includes 33 species/ subspecies accounting for 44\%, Bacillatoriophyta 18 species/ subspecies representing 24\%, Cyanophyta 12 species/ subspecies accounting for $16 \%$, Euglenophyta 11 species / subspecies accounting for $15 \%$, and the least is Pyrrhophyta with 1 species accounting for $1 \%$.

The wide distribution in the Xuanhuong Lake is not heterogeneous (Table 3). The composition of algae in the middle of the Lake and along the Lake is a discrepancy. Some algae species are present near the shore but not in the middle of the lake such as Coelastrum microporum and some species in the Scenedesmus genus and vice versa such as some species of the Chlorella genus...

\begin{tabular}{|c|c|c|}
\hline No. & Location & Quantity of algae \\
\hline 01 & XHS1 & 32 \\
\hline 02 & XHS2 & 28 \\
\hline 03 & XHS3 & 30 \\
\hline 04 & XHS4 & 27 \\
\hline
\end{tabular}

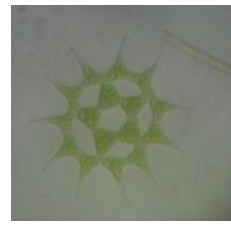

(a)

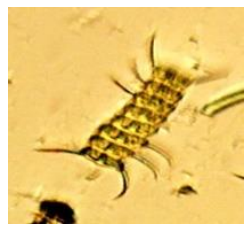

(b)

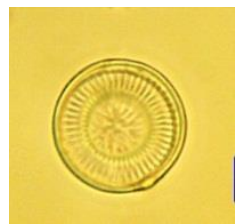

(c)

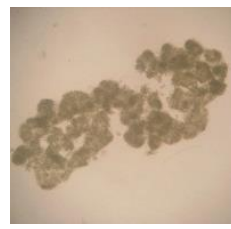

(d)

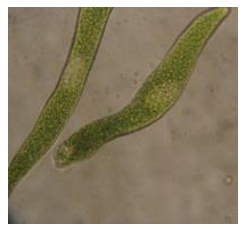

(e)

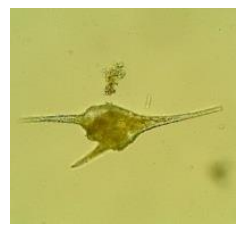

(f)

Figure 2. Photos of microalgae from Xuanhuong Lake

Note: (a) Pediastrum simplex var. duodenarium (Bailey) Rabenhorst; (b) Scenedesmus quadricauda var. armatus (Chodat.) Dedus; (c). Cyclotella sp; (d) Microcystic aeruginosa;

(e) Euglena oxyuris sp; (f) Ceratium hidrundinella.

During the survey process, it was recognized that a bloom of Mycrosistic repeatedly occurred especially in the location XHS3. This may be due to the penetration of XHS3 wastewater from lakeside restaurants and the influence of the wind blowing from the south to north as Mycrosistic densely concentrated at this location.

Among 75 identified species / subspecies there is only one representative of the Pyrrhophyta algae, a typical phylum of mountainous clean water. These algae's samples are collected at XH1 which is the main water supply to the lake. Also in the water also 
appeared dirty indicator species of Euglena viridis, Euglena acus, Synedra ulna, which indicates that Xuanhuong Lake's water is mesosaprobic (medium dirty).

\section{CONCLUSION}

75 species / subspecies of 13 sets, 8 layers, 5 phyla: Cyanophyta, Chlorophyta, Bacillatoriophyta, Euglenophyta, Pyrrophyta were identified. Chlorophyta is the most abundant with 33 species / subspecies (44\%); the second is bacillatoriophyta with 18 species / subspecies, the third cyanophyta with 12 species / subspecies (16\%), the fourth euglenophyta 11 species / subspecies $(15 \%)$, and the least pyrrophyta (1\%).

Although cyanophyta is not the most abundant, it is the major cause of Xuanhuong Lake algae bloom during the survey time.

A number of dirty indicator species as Euglena viridis, Euglena acus, Synedra $u l n a$, etc. were discovered, indicating that Xuanhuong Lake water is at the level of mesosaprobic (medium dirty).

\section{REFERENCES}

Cole, K. M., \& Sheath, R. G. (1990). Biology of the red algae. New York: The Press Syndicate of the University of Cambridge.

Dương, Đ. T. (1996). Phân loại vi khuẩn lam ở Việt Nam. Hà Nội: Nhà xuất bản Nông nghiệp.

Dương, Đ. T., \& Võ, H. (1997). Tảo nước ngọt Việt Nam, Phân loại bộ tảo lục (Chlorococcales). Hà Nội: Nhà xuất bản Nông nghiệp.

Nguyễn, V. T. (2003). Đa dạng sinh học tảo trong thủy vục nội địa Việt Nam. Thành phố Hồ Chí Minh: Nhà xuất bản Nông nghiệp.

Phạm, H. H. (1972). Tảo học. Sài gòn: Nhà xuất bản Bộ Giáo Dục (Trung tâm học liệu).

Prescott, G. W. (1962). Algae of the Western Great Lakes area. Dubuque, Iowa: WM.C. Brown Company Publisher.

Smith, G. M. (1950). The freshwater algae of the United States. NewYork: McGrawHill book company.

Yamagishi, T. (1992). Palnkton Algae in Taiwan (Formosa). Tokyo: Uchida Rokakuho. 


\title{
ĐA DẠNG VỀ̀ THÀNH PHẦN LOÀI KHU HỆ TẢO Ở HỒ XUÂN HƯONG, ĐÀ LẠT
}

\author{
Nguyễn Thị Thùy Linh ${ }^{a^{*}}$, Lê Hà Thua
}

${ }^{a}$ Khoa Sinh họ, Trường Đại học Đà Lạt, Lâm Đồng, Việt Nam

Tác giả liên hệ:Email: linhntt@dlu.edu.vn

\section{Lịch sử bài báo}

Nhận ngày 02 tháng 06 năm 2016

Chỉnh sửa lần 01 ngày 02 tháng 07 năm 2016 | Chỉnh sửa lần 02 ngày 02 tháng 08 năm 2016

Chấp nhận đăng ngày 28 tháng 08 năm 2016

\section{Tóm tắt}

Hồ Xuân Hương được xem như trái tim của thành phố Đà Lạt. Qua khảo sát điều tra, định danh thành phần vi tảo ở hồ Xuân Hương - Đà Lạt cho thấy hệ thục vật nổi ở đây khá đa dạng và phong phú. Mẫu được thu tại 4 địa điểm khác nhau ở hồ trong thời gian tù tháng 9 năm 2012 đến tháng 1 năm 2013. Kết quả điều tra đã xác định được 75 loài/ duới loài thuộc 13 bộ, 8 lớp thuộc 5 ngành: Cyanophyta (tảo lam), Chlorophyta (tảo luc), Bacillatoriophyta (tảo silic), Euglenophyta (tảo mắt), Pyrrhophyta (tảo giáp). Ngành có nhiều loài nhất là tảo lục (33 loài/duới loài) chiếm 44\%., kế đến là tảo silic (18 loài/duới loài) chiếm $24 \%$, tảo lam (12 loài/duới loài) chiếm 16\%, tảo mắt (11 loài/duói loài) chiếm $15 \%$, và it nhất là tảo giáp (1 loài) chiếm 1\%. Đáng luu ý với sư xuất hiện một số loài chỉ thi độ bẩn nhu Euglena viridis, Euglena acus, synedra ulna báo động múc nuoóc hồ Xuân Hưong thuộc múc mesosaprobe.

Từ khóa: Chỉ thị sinh học; Đa dạng sinh học; Hồ Xuân Hương; Tảo học. 